\title{
TÉCNICA PARA MENSURAÇÃO OVARIANA POR VIDEOLAPAROSCOPIA EM AVES - MODELO EXPERIMENTAL - CODORNA-JAPONESA (Coturnix coturnix japonica)
}

(Technique for ovarian measurement of birds by videolaparoscopy - experimental model - japanese quail - Coturnix coturnix japonica)

Marcia Helena Martins de Albuquerque ${ }^{1}$, Rogerio Luizari Guedes ${ }^{1}$, Peterson Triches Dornbusch ${ }^{1}$, Marina Stival Andrade ${ }^{2}$, Paula Beatriz Mangini², Barbara Weiss Stadler ${ }^{2}$, Anna Julia Zilli Lech ${ }^{2}$, Rogerio Ribas Lange ${ }^{1}$

\footnotetext{
${ }^{1}$ Programa de Pós-graduação em Ciências Veterinárias, Universidade Federal do Paraná, Curitiba, Paraná, Brasil.

${ }^{2}$ Departamento de Medicina Veterinária. Setor de Ciências Agrárias. Universidade Federal do Paraná, Curitiba, Paraná, Brasil.
}

Correspondência: rrlange@ufpr.br

RESUMO: A redução do tamanho e da vascularização do aparelho reprodutor feminino pode auxiliar no tratamento de aves de estimação que sofrem com postura crônica e outras complicações reprodutivas e facilitar a salpingohisterectomia. Usando como modelo a codorna-japonesa (Coturnix coturnix japonica), os objetivos do presente estudo foram a obtenção de um método eficaz para realizar a mensuração ovariana por videolaparoscopia de aves em postura e avaliar qual a redução proporcionada pela restrição luminosa por um período 24 dias. Foram realizadas videolaparoscopias em 18 codornas para a mensuração ovariana por meio das imagens obtidas utilizando uma régua milimétrica especialmente confeccionada e o software ImageJ. Após, receberem oito horas diárias de luz durante 24 dias, as aves foram submetidas à eutanásia e necropsia para nova mensuração ovariana. Dezesseis codornas apresentaram folículos ovarianos excedendo o campo visual do endoscópio. A régua milimétrica e o software ImageJ foram considerados eficazes para realizar a mensuração ovariana por videolaparoscopia quando os ovários não excedem o tamanho do campo visual, porém a técnica não é indicada para a mensuração de estruturas grandes como o ovário com pleno desenvolvimento folicular. Para utilizar a técnica descrita, é necessário desenvolver um acesso cirúrgico que permita a visualização do ovário em um único campo visual em todas as fases reprodutivas.

Palavras-chave: Ave; fotoperíodo; gônada; ovário; videocirurgia.

ABSTRACT: The reduction of the reproductive tract size and vascularization can assist the treatment of pet birds that suffer with chronic egg laying and other reproductive complications and facilitate a salpingohysterectomy. Using as model the Japanese Quail (Coturnix coturnix japonica), the objectives of this study were to obtain an effective method to perform the ovarian measurement of laying birds during the videolaparoscopy and evaluate the ovarian reduction originated with the photoperiod reduction for 24 days. Eighteen quails were submitted to videolaparoscopy to measure the ovarian size through the images obtained with a specially made millimetric ruler and the ImageJ software. The birds received eight hours of light per day for 24 days and then were euthanized and necropsied to remeasurement of ovarian size. Sixteen quails had big ovarian follicles that exceed the endoscope visual field. The millimetric ruler and the ImageJ software were 
considered effective to carry out the ovarian measurement using videolaparoscopy when the ovaries did not exceed the endoscope visual field, however this technique is not indicated to measure big organs such as the ovarian follicles in development. To use the technique described, it's necessary to promote a chirurgical access that allows the fully visualization of the ovary in different stages of the reproductive cycle in a single field of view.

Key Words: Avian; gonad; ovary; photoperiod; videosurgery.

\section{INTRODUÇÃO}

O estímulo externo que exerce maior influência no ciclo reprodutivo das aves é a luminosidade (OLANREWAJU et al., 2006; PEREIRA, 2014). Durante os períodos de maior luminosidade a massa total do ovário das aves aumenta em pelo menos três vezes e a massa do oviduto em dezesseis vezes (FOLLETT et al., 1973), sendo que estudos mais recentes relatam aumento da massa ovariana em até 175 vezes (PEREIRA, 2014). Um dos objetivos do presente estudo foi avaliar 0 comportamento do ovário das aves com redução de luminosidade por 24 dias, comparando o tamanho do ovário em fase de postura por meio de videolaparoscopia e após a restrição luminosa por necropsia. Essa redução pode auxiliar no tratamento de aves de estimação que sofrem com postura crônica, retenção de ovos e outras complicações reprodutivas, bem como facilitar a cirurgia de salpingohisterectomia, recomendada nestes casos (PYE et al., 2001), por reduzir não apenas o tamanho, mas também a vascularização (BOWLES, 2002; ECHOLS, 2002).

O principal objetivo deste estudo foi a obtenção de um método eficaz para realizar a mensuração do ovário durante a videolaparoscopia de aves considerando a magnificação proporcionada pela lente do endoscópio, usando como modelo a codornajaponesa (Coturnix coturnix japonica) e, assim, conseguir comparar o tamanho do ovário de um mesmo indivíduo em diferentes fases do ciclo reprodutivo.

\section{MATERIAL E MÉTODOS}

O estudo foi aprovado pelo Comitê de Ética no Uso de Animais do Setor de Ciências Agrárias da Universidade Federal do Paraná (Número 10/2014). Foram realizadas videolaparoscopias em codornas-japonesas (Coturnix coturnix japonica) com idade aproximada de dois anos, saudáveis, em postura, por um período de 30 dias.

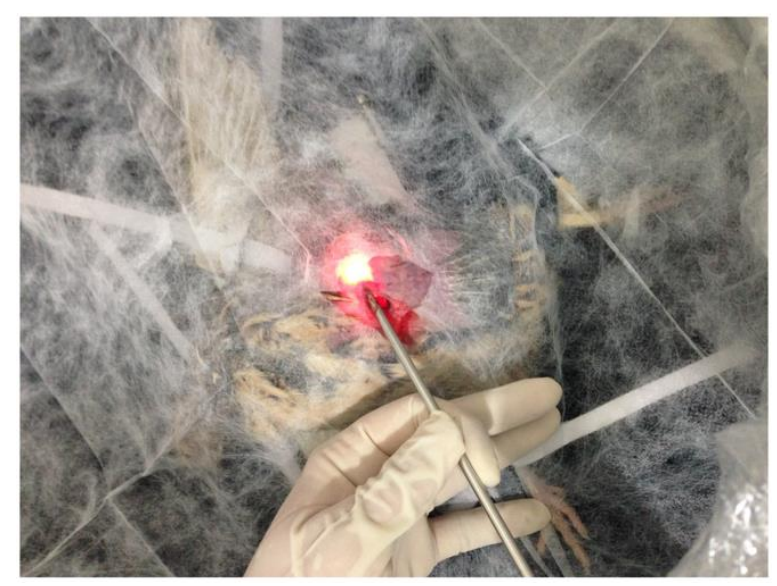

Figura 1 - Aspecto do posicionamento da codorna-japonesa (Coturnix coturnix japonica) e inserção do endoscópio rígido para a videolaparoscopia.

Por meio da mesma incisão, ventralmente, foi efetuada a ruptura do saco aéreo abdominal esquerdo com uma pinça laparoscópica Mixter $2.8 \mathrm{~mm}$ de diâmetro e $20 \mathrm{~cm}$ de comprimento, confeccionada sob medida para uso em animais de pequeno porte. Após a visualização do ovário, a pinça foi removida para a introdução de uma 
régua milimétrica de aço inox, posicionada paralelamente ao ovário a fim de permitir a captação de imagens para sua mensuração com o software ImageJ (NIH Image, Maryland, Estados Unidos).

A régua milimétrica de aço inox foi confeccionada especialmente para este experimento. Mede de comprimento 20 $\mathrm{cm}$, de largura em sua extremidade 2,0 $\mathrm{mm}$, em sua base $4 \mathrm{~mm}$ e possui espessura de 0,6 mm (Figura 2). Foi removida qualquer borda cortante a fim de evitar danos teciduais e seus $4,0 \mathrm{~mm}$ finais foram angulados em $90^{\circ} \mathrm{com} 0$ propósito de permitir o seu posicionamento paralelo às estruturas a serem mensuradas. A imagem obtida com a presença da régua posicionada paralelamente ao ovário foi utilizada para mensurar o ovário com o auxílio do software ImageJ. Após a retirada dos instrumentos, a musculatura foi suturada com poliglactina 910 (Bioline Fios Cirúrgicos, Goiânia, Brasil) em padrão interrompido simples, seguida da sutura da pele com o mesmo padrão e material.

Figura 2 - Régua milimétrica de aço inox com 20 $\mathrm{cm}$ de comprimento, 0,6 $\mathrm{mm}$ de espessura, $2 \mathrm{~mm}$ de largura na extremidade e $4 \mathrm{~mm}$ na base com seus $4 \mathrm{~mm}$ finais angulados.

O software ImageJ permitiu a inserção de uma medida conhecida de um milímetro, a qual foi obtida da imagem da régua milimétrica, para aferir as medidas desconhecidas do ovário na mesma imagem. Foram realizadas as medições do ovário quanto ao seu comprimento, largura, área e perímetro. O comprimento ovariano foi obtido da extremidade cranial do ovário até a extremidade caudal, sendo a sua extremidade cranial definida como aquela mais próxima ao pulmão e a extremidade caudal a oposta. A largura ovariana foi obtida por meio da mensuração da distância entre suas margens lateral e medial, sendo a margem lateral aquela mais próxima ao rim esquerdo e a medial a oposta. A altura do ovário foi obtida por meio da mensuração da distância entre a face dorsal e a face cranial do ovário. A área e o perímetro foram calculados pelo software ImageJ delimitando-se toda a extensão do ovário visualizada na imagem.

As aves de todos os grupos receberam cetoprofeno $2 \mathrm{mg} / \mathrm{Kg}$ I.M. a cada $24 \mathrm{~h}$ (Ketofen 1\%, Merial, Estados Unidos) durante três dias (HUEZA, 2008) após a videolaparoscopia, para assepsia da ferida cirúrgica foi utilizada iodopovidona duas vezes ao dia durante sete dias após o procedimento ou até completa cicatrização.

Após os procedimentos cirúrgicos, as aves receberam oito horas diárias de luz durante 24 dias. O tempo de iluminação da sala foi controlado por um temporizador digital automático FDD60/SB2 (G20, Santa Catarina, Brasil). A sala foi isolada completamente de luz natural e a iluminação artificial foi proveniente de quatro unidades de lâmpadas fluorescentes tubulares de 40W. Ao final dos 24 dias, todas as aves foram submetidas à eutanásia e necropsia.

A mensuração da luminosidade foi realizada em diferentes dias com 0 aplicativo para IOS Luxmeter (Application Manufactory UG, Brandenburg, Alemanha) com o aparelho posicionado em direção à luz dentro de diferentes gaiolas no nível da cabeça das aves. No período em que a luz se encontrava acesa, houve variação da luminosidade entre 220 e 270 lux. No período em que a luz permaneceu apagada, a mensuração foi sempre de zero lux. 


\section{RESULTADOS}

O tempo médio para a realização da videolaparoscopia das codornas foi de 18,3 minutos por ave. Das 18 codornas que estavam em postura, apenas duas apresentaram ovário de tamanho compatível com o campo visual do endoscópio (Figura 3).

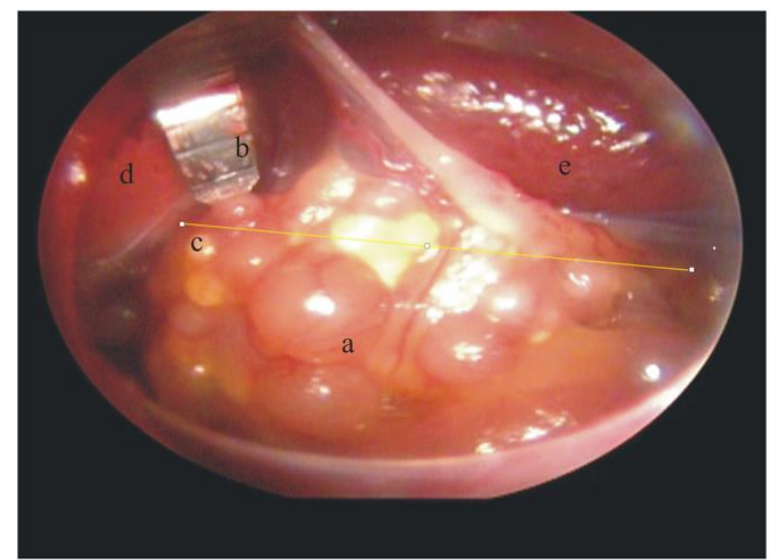

Figura 3 - Imagem obtida por meio de videolaparoscopia de uma fêmea de codornajaponesa (Coturnix coturnix japonica), mostrando o ovário (a), a régua milimétrica (b), o comprimento do ovário (linha amarela indicada pela letra c) obtido por meio do software imagej, o pulmão esquerdo (d) e o rim esquerdo (e).

As outras $16 \quad$ codornas apresentaram uma das seguintes situações: um folículo amarelo grande obstruindo a visualização de qualquer limite ovariano (Figura 4) ou o comprimento do ovário (com seus folículos) mais longo que o campo visual, impossibilitando a visualização das extremidades cranial e caudal do mesmo (Figura 5).

Em onze codornas a totalidade do campo visual era ocupada por parte do ovário.

Foi observado por meio da videolaparoscopia um caso com um único grande folículo amarelo (em desenvolvimento), até 11 folículos em 16 codornas, e em apenas duas o ovário apresentava-se quiescente.
Quatorze codornas cessaram a postura entre três e quatro dias após a videolaparoscopia. Três aves voltaram a postura após 14 a 15 dias da cirurgia e uma apresentou um ovo no momento da necropsia aos 24 dias do início do experimento. $O$ tempo médio para cicatrização completa da ferida cirúrgica foi de nove dias.

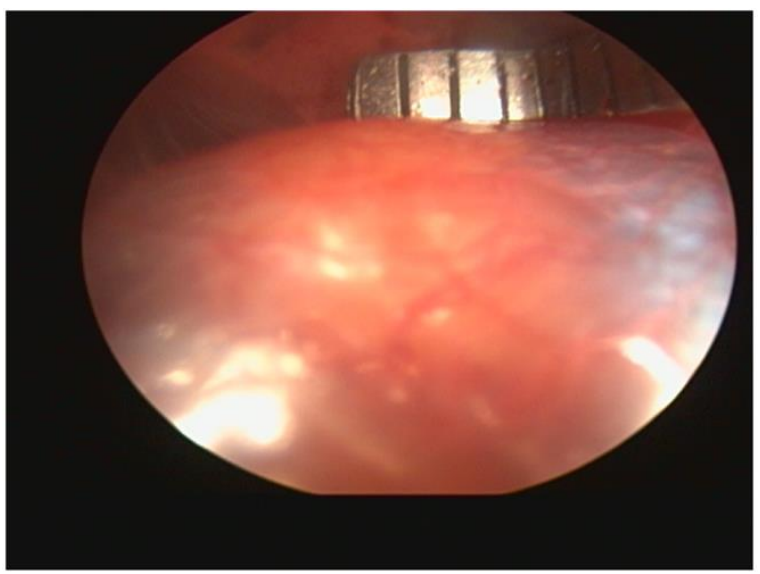

Figura 4 - Imagem obtida por videolaparoscopia de uma fêmea de codorna-japonesa (coturnix coturnix japonica), mostrando um grande folículo amarelo na lateral esquerda do ovário, obstruindo a visualização de todos os limites ovarianos.

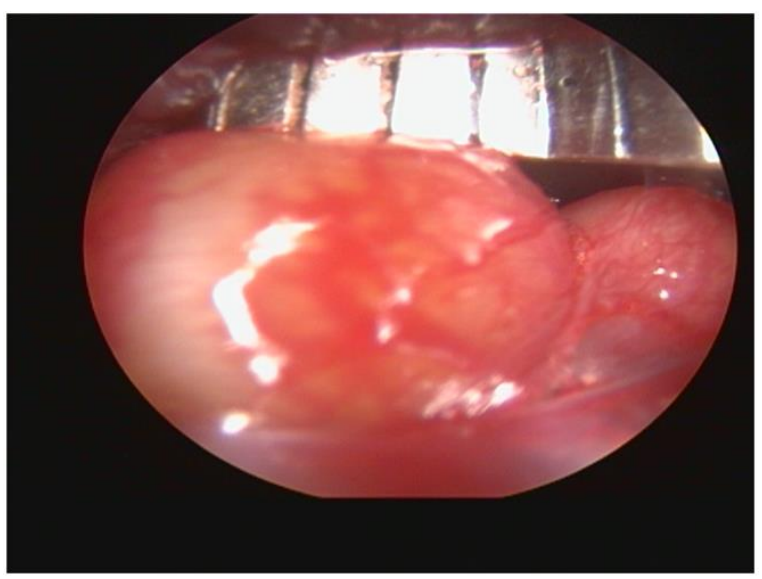

Figura 5 - Imagem obtida por videolaparoscopia de uma fêmea de codorna-japonesa (coturnix coturnix japonica), mostrando dois grandes folículos amarelos. Neste indivíduo, haviam mais folículos, cranial e caudalmente à imagem, não sendo possível a visualização completa do contorno ovariano, impedindo a mensuração do seu comprimento.

$\mathrm{Na}$ necropsia foi observado que não houve danos ao ovário ou aos órgãos próximos ao acesso, como rim e 
pulmão esquerdos e houve cicatrização completa do saco aéreo abdominal esquerdo. As medidas obtidas dos ovários avaliados foram: comprimento $18,79 \pm 8,86 \mathrm{~mm}$; largura 10,40 $\pm 6,12$ $\mathrm{mm}$; área 173,28 $\pm 201,75 \mathrm{~mm}^{2}$; e perímetro $53,82 \pm 32,13 \mathrm{~mm}$.

\section{DISCUSSÃO}

O tempo médio para a realização das videolaparoscopias pode ser considerado longo, pois excede o demandado por algumas cirurgias de salpingohisterectomia (PYE et al., 2001). Isso pode ser explicado pela inexperiência do cirurgião e do assistente, visto que o tempo diminuiu ao longo do experimento. A dificuldade de posicionamento do endoscópio para visualizar completamente o ovário também demandou tempo, pois em todos os animais sempre era realizada a tentativa de se visualizar o máximo possível do órgão, porém na maioria dos casos sem êxito.

A técnica cirúrgica descrita mostrou-se útil para a avaliação do ovário de codornas-japonesas com folículos pequenos ao permitir a visualização completa do órgão e por demonstrar, à necropsia, que não houve danos ao ovário ou aos órgãos adjacentes, os quais poderiam ser ocasionados tanto pelo endoscópio como pela régua. No entanto, não possibilitou a avaliação ovariana quando havia um grande folículo amarelo de tamanho superior ao estroma ovariano na região lateral do ovário, situação na qual o campo visual do endoscópio é inteiramente tomado apenas pela imagem total ou parcial do folículo. O sítio de incisão adotado para a videolaparoscopia foi semelhante ao utilizado em técnicas de celiotomia ou endoscopia com incisão única (PYE et al., 2001; CLAYTON; RITZMAN, 2006), porém diferiu do utilizado pela maioria dos autores em técnicas de celiotomia ou salpingohisterectomia por videocirurgia (ECHOLS, 2002; HERNANDEZ-DIVERS et al., 2007; DIVERS, 2010).

A régua milimétrica e o software ImageJ foram considerados eficazes para realizar a mensuração ovariana por videolaparoscopia quando os ovários não excedem o tamanho do campo visual, porém é necessário que a estrutura a ser mensurada, neste caso o ovário, sempre esteja posicionada paralelamente à régua. $O$ mesmo padrão foi utilizado para realizar as medidas durante a necropsia a fim de minimizar possíveis erros ao empregar diferentes instrumentos métricos. Desta maneira, as medidas aferidas por imagem obtida com a presença da régua milimétrica $e$ analisadas pelo software ImageJ foram similares às de mensuração ovariana direta na necropsia. No entanto, nas situações em que os grandes folículos amarelos estão situados na margem esquerda do ovário e obstruem o campo visual, a mensuração mostrou-se inconsistente por não permitir a definição de todos os seus limites (cranial, caudal e laterais). Nas situações em que um folículo estava situado nas regiões cranial ou caudal do ovário, foi possível com o endoscópio avaliar grande parte do ovário, porém na maioria dos casos não foi possível compreender todo o órgão dentro do mesmo campo visual, portanto não permitindo a mensuração com a régua milimétrica e o software. Outro estudo já foi realizado para avaliar um instrumento confeccionado para mensuração de estruturas por endoscopia, porém com 0 intuito de determinar apenas 0 diâmetro das mesmas (LI et al., 2015).

A dificuldade de visualização completa do ovário se deve à pequena distância entre o órgão e o endoscópio, pois não há profundidade suficiente que permita o afastamento do instrumento a fim de ampliar o campo visual. São necessários outros estudos com diferentes acessos cirúrgicos para a 
videolaparoscopia, buscando maior distância do ovário para visualização ovariana completa mesmo com folículos ovarianos bem desenvolvidos, aceitando assim a mensuração por meio da régua milimétrica e do software ImageJ. Outra alternativa seria a repetição dessa pesquisa com o uso de lentes de grande ângulo visual a fim de avaliar se é possível realizar a mensuração ovariana com auxílio da régua milimétrica. Uma das maiores limitações para a endoscopia é o pequeno campo visual, no entanto lentes de ângulo amplo estão atualmente em uso na medicina e na veterinária para ampliar o campo visual do endoscópio e novas tecnologias estão surgindo para diminuir ou anular a distorção da imagem (ROULET et al., 2010).

Em aves como galinhas e avestruzes foi possível realizar uma adequada avaliação ovariana utilizando ultrassonografia, porém a mesma imagem também não consegue compreender todos os folículos (MELNYCHUK et al., 2002; PEZOA POBLETE et al., 2013), não permitindo sua mensuração adequada por este instrumento. Em peixes, a mensuração de ovos por ultrassonografia subestimou em 52\% o tamanho dos mesmos (BRYAN et al., 2007).

O fotoperíodo utilizado para a restrição luminosa foi de oito horas de luz por dia, o que corrobora com o descrito por diversos autores que citam que 0 fotoperíodo utilizado para reduzir a atividade reprodutiva é de oito a dez horas de luz diárias (BOWLES, 2002; ROSEN, 2012; PEREIRA, 2014).

O presente estudo teve como objetivo principal obter medidas ovarianas pareadas, ou seja, da mesma ave antes e após a restrição luminosa, visto que nenhum dos estudos prévios que avaliou a proporção de aumento do tamanho do aparelho reprodutor de aves em períodos de menor e maior luminosidade realizou comparações pareadas, 0 que determinaria maior precisão dos dados. As medidas ovarianas antes da restrição luminosa foram realizadas por meio de videolaparoscopia e após a restrição por necropsia. A necropsia, portanto, somente estava prevista para ser realizada ao final dos 24 dias de fotoperíodo reduzido. Novos estudos são necessários para proceder a aferição do ovário por meio de videolaparoscopia, utilizando a régua milimétrica e o software ImageJ, realizando a eutanásia dos animais em seguida para aferir diretamente o ovário e realizar as comparações, ou ainda utilizando novos acessos cirúrgicos ou lentes de grande ângulo visual.

\section{CONCLUSÃO}

Considerando-se as codornasjaponesas, os resultados mostram que a videolaparoscopia não é indicada para a mensuração do ovário em pleno desenvolvimento folicular. São necessários novos estudos para desenvolver uma técnica que permita a visualização do ovário em um único campo visual em todas as fases reprodutivas a fim de permitir a mensuração.

\section{REFERENCIAS}

BOWLES, H. L. Reproductive diseases of pet bird species. The veterinary clinics of North America. Exotic animal practice, Philadelphia, v.5, n.3, p.489-506, 2002.

BRYAN, J. L. et al. Estimation of gonad volume, fecundity, and reproductive stage of shovelnose sturgeon using sonography and endoscopy with application to the endangered pallid sturgeon. Journal of Applied Ichthyology, Hoboken, v.23, n.4, p.411-419, 2007.

CARPENTER, J. W. Exotic Animal Formulary. 3.ed. St. Louis: Elsevier Saunders, 2005. 
CLAYTON, L.; RITZMAN, T. Egg Binding in a Cockatiel (Nymphicus hollandicus). The Veterinary Clinics of North America. Exotic Animal Practice, v.9, n.3, p.511-518, 2006.

DIVERS, S. Avian Endosurgery. The veterinary clinics of North America. Exotic animal practice, Philadelphia, v.13, n.2, p.203-216, 2010.

ECHOLS, M. S. Surgery of the Avian Reproductive Tract. Seminars in Avian and Exotic Pet Medicine, v.11, n.4, p.177-195, 2002.

FOLLETT, B. K. et al. The influence of photoperiod on nest building, ovarian development and luteinizing hormone secretion in canaries (Serinus canarius). Journal of Endocrinology, v.59, p.151162, 1973.

HERNANDEZ-DIVERS, S. et al. Endoscopic Orchidectomy and Salpingohysterectomy of Pigeons (Columba livia): An Avian Model for Minimally Invasive Endosurgery. Journal of Avian Medicine and Surgery, v.21, n.1, p.22-37, 2007.

HESS, L. The Ethics of Exotic Animal Analgesia. Journal of Avian Medicine and Surgery, v.24, n.1, p.72-76, 2010.

HUEZA, I. M. Avian pharmacology: employing anti-inflammatory drugs in wild birds. ARS Veterinária, v.24, n.1, p.1524, 2008.

OLANREWAJU, H. A. et al. A review of Lighting Programs for Broiler Production. International Journal of Poultry Science, v.5, n.4, p.301-308, 2006.

PEREIRA, R. J. G. Reprodução das Aves. In: CUBAS, Z. S.; SILVA, J. C. R.; CATÃO-DIAS, J. L. (Eds). Tratado de Medicina de Animais Selvagens. $2^{\circ}$ ed. São Paulo: Roca, 2014. p.2235-2269.

PEZOA POBLETE, P. C. et al. Atividade folicular ovariana em avestruz (Struthio camelus) avaliada por ultrassonografia e sua relação com fotoperíodo e postura. Arquivo Brasileiro de Medicina Veterinária e Zootecnia, v.65, n.5, p.1573-1576, 2013. PYE, G. W. et al. Endoscopic Salpingohysterectomy of Juvenile Cockatiels (Nymphicus hollandicus). Journal of Avian Medicine and Surgery, v. 15, n.2, p.90-94, 2001.

ROSEN, L. B. Avian Reproductive Disorders. Journal of Exotic Pet Medicine, v.21, n.2, p.124-131, 2012.

LI, Z. et al. Endoscopic measurement of variceal diameter. World Journal of Gastroenterology v.21, n.7, p.21402146, 2015.

MELNYCHUK, V. L. et al. Use of ultrasonography to characterize ovarian status in chicken. Poultry science, v.81, n.6, p.892-895, 2002.

ROULET, P. et al. $360^{\circ}$ Endoscopy Using Panomorph Lens Technology. Endoscopic Microscopy V, v. 7558, 2010. 